\title{
Title: Evaluating the performance of neutrality tests of a local community using a niche-structured simulation model
}

Running title: Performance of neutrality tests

Authors: Yayoi Takeuchi ${ }^{1, *}$ and Hideki Innan ${ }^{2}$

${ }^{1}$ Center for Environmental Biology and Ecosystem Studies, National Institute for Environmental Studies, Tsukuba, Ibaraki, 305-8506, Japan ${ }^{2}$ Graduate University for Advanced Studies, Hayama, Kanagawa 240-0193, Japan

${ }^{*}$ Corresponding author: e-mail takeuchi.yayoi@nies.go.jp 


\section{Abstract}

2 Understanding the processes that underlie the species diversity and abun3 dance in a community is a fundamental issue in community ecology. While 4 the species abundance distributions (SADs) of various natural communities 5 may be well explained by Hubbell's neutral model, it has been repeatedly 6 pointed out that Hubbell's SAD-fitting approach lacks power to detect the 7 effects of non-neutral factors such as niche differentiation, but our under8 standing on its quantitative effect is limited. Here, we conducted extensive 9 simulations to quantitatively evaluate the performance of the SAD-fitting method and other recently developed tests. For the simulations, we developed a new niche model that incorporates both random stochastic demography of individuals and non-random replacements of individuals, i.e. niche differentiation. It allows us to explore situations with various degrees of niche differentiation. We found that niche differentiation has strong effects on the $\mathrm{SAD}$ and the number of species in the community under this model. We then examined the performance of neutrality tests including Hubbell's SADfitting method using the extensive simulations. It was demonstrated that all these tests have relatively poor performance except for the cases with very strong niche-structure, as has been pointed out by previous studies. This should be because two important parameters in Hubbell's model are usually unknown, and are commonly estimated from the data to be tested. To demonstrate this point, we showed that the precise estimation of the two parameters substantially improved the performance of these neutrality tests, indicating that poor performance of neutrality tests can be caused by overfitting of Hubbell's neutral model with unrealistic parameters. Our results 
26 emphasize the importance of accurate parameter estimation, which should

27 be estimated from data independent from the local community to be tested.

28 Keywords

${ }_{29} \quad$ Exact test; Lognormal; Logseries; Model fitting; (\#3-1)Neutral theory;

30 Species-abundance distribution, Species richness; Stochasticity 
Introduction

Ecological communities in nature comprise complex consortia of species with intricate structure; in a tropical forest, for instance, over a thousand tree species co-exist in one area (Condit et al., 2006). One of the major aims in community ecology is to understand the processes that underlie the species diversity and abundance in a community (Tilman, 1982; Lande et al., 2003). Community ecologists have developed a number of models to explore community structure, and the fit of these models to empirical community data have been examined. The species abundance distribution (SAD) is a basic metric to describe the relative abundance of species in a community, and observed SADs were often used for testing these theoretical models (Fisher et al., 1943; Preston, 1948; Tokeshi, 1990; Hubbell, 2001; Ulrich et al., 2010; Locey and White, 2013).

Two major categories of theories have been developed to explain the data of community structure; the niche theory incorporates deterministic factors such as inter-species competition and niche differentiation while some models allow stochastic (random) process. The other is the neutral theory, which considers random drift as the major player in community composition without including any deterministic factor. Traditionally, deterministic factors have been considered to play a major role to shape the species composition and diversity in a community (Tilman, 1982; Tokeshi, 1990, 1992; Chesson, 2000; Sugihara et al., 2003). Niche theories assume that each species in a community would be specialized to particular combinations of resources through inter-species competition (Westoby et al., 2002). This competition involves a number of deterministic factors including tradeoffs, and as a consequence, 
it drives interaction between species, thereby resulting in the co-existence of multiple species at equilibrium. Niche models are widely accepted because there are a number of field observations exhibiting clear evidence for niche differentiation (Wright, 2002). In addition, theories under niche models predicts that SAD should be approximated by a lognormal distribution, and this prediction is consistent with many field observations (Tilman, 1982; Tokeshi, 1990, 1992; Sugihara et al., 2003; Harpole and Tilman, 2006).

On the other hand, the neutral theories have also been advocated in the last decade. Caswell (1976) firstly introduced three neutral models into ecology but they were not well accepted in the 20th century because they failed to provide a good fit to data from to natural communities. Hubbell's neutral model (Hubbell, 2001) changed the situation; as the model was found to provide a good fit to a wide range of empirical observations. His model assumes that all individuals are ecologically or functionally equivalent, i.e., no difference in reproduction and mortality among individuals. Thus, the composition of a local community is determined only by stochastic extinction, local birth and dispersal from the nested metacommunity with random speciation. (\#3-4)This process is elegantly summarized by only three parameters, the fundamental diversity number $(\theta)$, the migration rate $(m)$ from the metacommunity to the local community and the number of individuals in the local community $(J)$, and the shape of the expected SAD in the local community can be characterized by a function of $\theta, m$ and $J$. (\#3-3) The distribution derived from the neutral model is so-called zero-sum multinomial distribution. This very simple model can be considered to be one of the most strict forms of neutral models with a number of simplified assumptions. 
Despite these strict assumptions, the fit of Hubbell's neutral model to field data seems to be quite good; SADs from a wide range of communities were very well explained by Hubbell's neutral model (e.g., tropical forests (Etienne, 2005; Volkov et al., 2007), fishes (Etienne and Olff, 2005), and birds (He, 2005)).

This good performance of Hubbell's neutral model is particularly surprising because (i) it provides a good fit to data from tropical forests (Etienne, 2005; Volkov et al., 2007), in which it has been believed that niche differentiation would be the major force to maintain high species diversity (Wright, 2002), (ii) Hubbell's neutral model sometimes shows a better fit (particularly in the abundance of rare species) than those predicted by deterministic models (Volkov et al., 2005; He, 2005).

The historical reason behind the rise of Hubbell's neutral model was partly because of the increase of sample size. When SAD was typically obtained from a small number of individuals from a community, such a SAD was well-fitted by a lognormal distribution (Preston, 1948) or even a logseries distribution (Fisher et al., 1943). Preston $(1948,1962)$ firstly predicted that if the sample size of a community was large enough, a SAD would be a symmetric distribution, i.e., lognormal. However, the situation has changed when community data with a large sample size in a closed community became available, e.g., 50-ha forest dynamics plots of Smithsonian tropical research institute. It was found that such SADs are negatively skewed with a large excess of rare species over the prediction made by the lognormal model. Hubbell's neutral model fitted to these rare species better and thus the model became popular even though assumptions of the underlying theory were difficult to 
accept for some ecologists. His model has been used as a first null model to be tested, which was formally suggested in a recent review by Alonso et al. (2006) (but see Gotelli and McGill, 2006). Meanwhile, lognormal and logseries distributions became alternative SADs that represent some nonneutral process as already demonstrated by theoretical studies (May, 1975; Sugihara, 1980; Engen and Lande, 1996; Magurran, 2004).

There has been a great deal of debate on the interpretation of the good-fit of Hubbell's neutral model. As it is obvious that Hubbell's neutral model cannot be the exclusive explanation, his neutral model has been challenged by a number of authors. Several studies demonstrated that non-neutral models fit to observed SADs better than Hubbell's neutral model, e.g., in grassland communities (Harpole and Tilman, 2006), coral reefs (Dornelas et al., 2006), tropical forests (Etienne, 2005), aphids (He, 2005) and fishes (He, 2005). Technical problems in the interpretation of fitting Hubbell's neutral model to field data have been debated so far. One is that Hubbell's neutral model is so flexible that it can predict SADs that are generated by nonneutral models (Adler et al., 2007; Chave, 2004; Bell, 2005; Chisholm and Pacala, 2010). This is because Hubbell's neutral model predicts the SAD in the local community of interest conditional on $\theta$ and $m$, which are usually unknown. Therefore, in the fitting process, $\theta$ and $m$ are conventionally estimated from the data of the "local" community to be tested. As these two estimated parameters are optimized to the local community, it is not surprising that Hubbell's neutral model often fits the observed SAD. Consistent with this intuitive understanding, there are a number of theoretical reports demonstrating that non-neutral models can predict very similar patterns of 
SAD and other summary statistics to those expected under Hubbell's neutral model. For example, Chisholm and Pacala (2010) have recently presented an analytical framework to prove that niche-structure could predict a similar pattern of SADs of neutral communities (see also Purves and Pacala, 2005). Together with other demonstrations under various conditions, the consensus seems to be that niche and neutral models can generate similar patterns if parameters are adjusted (Adler et al., 2007; Chave, 2004; Volkov et al., 2005; Bell, 2005). It is therefore apparent that the major problem is that the SAD-fitting approach of Hubbell's neutral model (2001) likely misses the signature of non-neutral factors. Thus, it is clear that the SAD-fitting generally has low power to reject neutrality, as has been pointed out repeatedly (Adler et al., 2007; Chave, 2004; Bell, 2005; Chisholm and Pacala, 2010; Clark, 2012; Rosindell et al., 2012), but there has not been a systematic likelihood-based quantitative test of this. For example, Chave et al. (2002) visually compared SADs generated from neutral and niche models, but they did not provide statistical tests of the neutral model.

Motivated by this problem in the SAD-fitting approach of Hubbell's neutral model, other kinds of statistical methods have recently been developed. One is the "exact test" proposed by Etienne (2007). The idea is based on Fisher's exact test, and similar tests hlare also introduced in population genetics by Slatkin $(1994 ; 1996)$ (see also Innan et al., 2005). It should be noted that one cannot expect the "exact" performance of this test because it also requires estimated values of $\theta$ and $m$ (Etienne, 2007), so that the same problem as the SAD-fitting still remains. Furthermore, because the "exact" computation of the probabilities of 
all possible patterns of species abundance is not computationally feasible, it employs approximate treatments using likelihood.

Another approach to fit the neutral model is summary statistic-based tests similar to Watterson's homozygosity test in population genetics. Shannon's index in ecology is essentially identical to homozygosity in population genetics. Jabot and Chave (2011) developed a statistical test, to examine if the observed Shannon's index is consistent with a null distribution predicted by Hubbell's neutral model conditional on the number of observed species. Again, it requires estimated values of $\theta$ and $m$. Because these tests are relatively new and their applications to real field data are still limited, it is also unclear how they perform under what conditions.

The main aim of this work is to evaluate the performance of these neutrality tests quantitatively by extensive simulations. For this purpose, we first develop a simple niche model, which incorporates stochastic demography. The advantage of this model is that it has a parameter, $p$, which represents the degree of niche differentiation. $p$ is given by the closed interval $[0,1]$; when $p=1$, the model is identical to Hubbell's neutral model, and as $p$ decreases, the degree of niche differentiation becomes stronger. In the extreme case with $p=0$, it is assumed that each niche can be occupied by only one particular species. This idea of niche differentiation is similar to some of the previous studies(Gravel et al., 2006; Tilman, 2004); their models consider a stochastic process of death and birth, in which each species is assumed to have a preference to a specific environment, i.e., niche. As with our model, these models have a parameter to determine the degree of niche overlap among species. Thus, with this type of neutral-niche model, we can quantitatively assess 
the relationship between the degree of niche differentiation (i.e., $p$ ) and the performance of various neutrality tests.

In this work, by performing extensive simulations with $p$ changing from 1 to 0 , we explore the performance of various neutrality tests. We include Hubbell's SAD-fitting approach (Hubbell, 2001), Etienne's exact test (Etienne, 2007) and summary statistic-based tests, including those using Shannon's index (Jabot and Chave, 2011). In addition, we also develop similar tests using other summary statistics, and their performances are compared in various conditions. We also discuss the possibility of more powerful approaches.

\section{Model}

(\#3-2) Our model is spatially implicit and we focus on the species abundance in a local community, while the spatially explicit neutral SAD models have been developed recently (Rosindell et al., 2008; Matthews and Whittaker, 2014). It is assumed that there is a metacommunity that provides a source of individuals for the local community. Let the metacommunity consist of $J_{M}$ individuals while there are $J$ individuals in the local community constantly. It is usually assumed that the size of the metacommunity is several orders of magnitude larger than the size of the local community.

\section{Hubbell's Neutral Model}

As our niche model is very similar to Hubbell's neutral model (Hubbell, 2001). except for one process, we first explain how a local community can be simulated under Hubbell's neutral model (Hubbell, 2001). Here, assumed 
227 in time. steps.

that we can count the number of individuals in a local community in the field, so we fix $J$. Thus, we consider that the neutral model has only two parameters, $\theta$ and $m$. Each simulation run can be described with the following

(i) Create the metacommunity: The diversity and relative abundance of species in the metacommunity are pre-determined by the composite parameter $\theta$ that is referred as the "fundamental biodiversity number" $\left(\theta=\frac{v}{1-v}\left(J_{M}-\right.\right.$ 1 ), where $v$ is the probability of speciation per birth). The configuration of the metacommunity is governed by Ewens sampling formula (Ewens, 1972) and its SAD follows a logseries distribution (Hubbell, 2001). For theoretical details, see Etienne and Alonso (2007). Under a given value of $\theta$, a random configuration of the metacommunity with $J_{M}$ individuals can be obtained by following Hubbell's method (2001) (see Appendix for detailed algorithms). Let $S_{M}$ be the total number of species in the simulated metacommunity. This configuration of the metacommunity will be fixed in the following steps for simulating the local community.

(ii) Create the initial local community: The initial state of the local community with $J$ cells is randomly created. That is, all $J$ cells are filled by randomly choosing individuals from the metacommunity. Conditional on this initial state, the dynamics of local community can be simulated forward 228 
(iii) Simulate the dynamics of the local community: Simulate the dynamics of the local community by randomly replacing individuals in the local community. The simulation can be performed by repeating a number of small time steps. At each time step, individuals die at a given mortality rate (all individuals have equal susceptibility to mortality). Empty cells due to deaths are randomly recolonized by immigrants from the metacommunity with probability $m$ and by offspring of the remaining local community members with probability $1-m$. Thus, there are no empty cells because a death is always replaced by either a birth or an immigrant (i.e., the "zero-sum dynamics" are applied). This demographic stochasticity is called "ecological drift"(Hubbell, 2001). Another important assumption is ecological equivalence among species or individuals, i.e., all individuals have equal mortality rates, equal fecundities, and equal probabilities of their offspring taking over the cell on which they land, regardless of the previous occupant of the cell.

(iv) Evaluate the configuration of the local community: The final simulation result of the local community is obtained by repeating 20,000 time steps. Then, the diversity and relative abundance of species in the local community can be evaluated.

\section{Niche Model}

In our niche model, we modify steps (ii) and (iii) of Hubbell's neutral model to incorporate the effect of niche differentiation in the local community. 
(ii) Create the initial local community: It is assumed that there are $N$ different niches in the local community. Each cell in the local community belongs to one of the $N$ niches, and the number of cells in each niche is determined by a multinomial distribution with parameters $\left(\frac{1}{N}, \frac{1}{N}, \frac{1}{N}, \ldots \frac{1}{N}\right)$. $N$ is determined such that it does not exceed the total number of species in the metacommunity, $S_{M}$, which was given in the previous step (i). $q_{i, j}$ is the parameter to specify the property of the $i$ th niche $(i=1,2,3, \ldots N)$, which is determined such that $q_{i, j}=1$ if the $i$ th niche allows the $j$ th species to occupy, otherwise $q_{i, j}=0$. Therefore, property of niche adaptation of of the entire local community is described by a $N \times S_{M}$ matrix denoted by $M$ :

$$
M=\left[\begin{array}{ccccccc}
q_{1,1} & q_{1,2} & q_{1,3} & q_{1,4} & q_{1,5} & \cdots & q_{1, S_{M}} \\
q_{2,1} & q_{2,2} & q_{2,3} & q_{2,4} & q_{2,5} & \cdots & q_{2, S_{M}} \\
q_{3,1} & q_{3,2} & q_{3,3} & q_{3,4} & q_{3,5} & \cdots & q_{3, S_{M}} \\
q_{4,1} & q_{4,2} & q_{4,3} & q_{4,4} & q_{4,5} & \cdots & q_{4, S_{M}} \\
q_{5,1} & q_{5,2} & q_{5,3} & q_{5,4} & q_{5,5} & \cdots & q_{5, S_{M}} \\
\vdots & \vdots & \vdots & \vdots & \vdots & \vdots & \vdots \\
q_{N, 1} & q_{N, 2} & q_{N, 3} & q_{N, 4} & q_{N, 5} & \cdots & q_{N, S_{M}}
\end{array}\right]
$$

We here introduce a parameter, $p$, which characterize the overall nichespecificity. Let us first consider the most strict niche differentiation case with $p=0$, in which we assume that there is a one-by-one relationship between niche and species. That is, the $i$ th niche can be occupied only by the 
$267 i$ th species, so that the matrix is given by

$$
M_{\mid p=0}=\left[\begin{array}{ccccccccc}
1 & 0 & 0 & 0 & 0 & \cdots & 0 & \cdots & 0 \\
0 & 1 & 0 & 0 & 0 & \cdots & 0 & \cdots & 0 \\
0 & 0 & 1 & 0 & 0 & \cdots & 0 & \cdots & 0 \\
0 & 0 & 0 & 1 & 0 & \cdots & 0 & \cdots & 0 \\
0 & 0 & 0 & 0 & 1 & \cdots & 0 & \cdots & 0 \\
\vdots & \vdots & \vdots & \vdots & \vdots & \vdots & \vdots & \vdots & \vdots \\
0 & 0 & 0 & 0 & 0 & \cdots & 1 & \cdots & 0
\end{array}\right]
$$

268 We here define $q_{i, i}=1(i=1,2,3, \ldots N)$ for convenience, so that the remain269 ing species (from species $N+1$ to $S_{M}$ ) cannot survive in any niche in the 270 local community.

271 On the other hand, in the other extreme case with $p=1$, it is assumed 272 that all niches can be occupied by any of the $S_{M}$ species, so that $M_{\mid p=1}$ is 273 given by

$$
M_{\mid p=1}=\left[\begin{array}{ccccccc}
1 & 1 & 1 & 1 & 1 & \cdots & 1 \\
1 & 1 & 1 & 1 & 1 & \cdots & 1 \\
1 & 1 & 1 & 1 & 1 & \cdots & 1 \\
1 & 1 & 1 & 1 & 1 & \cdots & 1 \\
1 & 1 & 1 & 1 & 1 & \cdots & 1 \\
\vdots & \vdots & \vdots & \vdots & \vdots & \vdots & \vdots \\
1 & 1 & 1 & 1 & 1 & \cdots & 1
\end{array}\right]
$$

${ }_{274}$ We here consider an intermediate case, where $p$ represents the expected 275 proportion of species that can occupy a niche. Let us define $\bar{q}_{i}$ as the pro- 
276

portion of species that be accepted in the $i$ th niche:

$$
\bar{q}_{i}=\sum_{j=1, i \neq j}^{S_{M}} \frac{q_{i, j}}{S_{M}-1} .
$$

${ }_{277}$ Then, $M_{\mid p}$ is given such that

$$
E\left(\bar{q}_{i}\right)=p
$$

holds for all rows.

For a simulation given a specified value of $p$, we can construct a random matrix $M_{\mid p}$ by defining a certain function for $\bar{q}_{i}$. Any function should work, and we here use a beta function $\operatorname{Bet} a(1, b)$, where $b$ is adjusted so that the mean of $\operatorname{Beta}(1, b)$ is $p$ (For example, $b=1$ given if $p=0.5$ ). A beta distribution provides a relatively wide range of values between 0 and 1 , so that the local community can consist of variety of niches, from strong to weak niches, with an intermediate $p$. Let $q_{i}^{\prime}$ be a random value from $\operatorname{Beta}(1, b)$. Then, $Q_{i}$, the number of species that are able to survive in the $i$ th niche, follows a binomial distribution, $\operatorname{Binom}\left(S_{M}-1, q_{i}^{\prime}\right)$. With $Q_{i}$, vector $\left\{q_{i, 1}, q_{i, 2}, q_{i, 3}, \ldots q_{i, S_{M}}\right\}$ can be constructed as follows. First, $q_{i, i}=1$ is given as defined. Next, $Q_{i}$ columns are randomly chosen from the remaining $S_{M}$ columns. By using this method, all row of the matrix $M$ can be determined.

The initial state of the local community can be created once this matrix $M$ is specified. Note that, as stated earlier, it is already determined which cells in the local community belong to which niches. With this setting, each of the $J$ empty cells is filled by the following procedure. For a cell that belongs to the $i$ th niche,

I, Pick a random individual from the metacommunity. Let $j$ be the species number of the chosen individual. 
II, Fill the cell if $q_{i, j}=1$, otherwise go to [I]. Continue until this cell is filled.

This initial setting is fixed through the following forward simulation of the local community. The configuration of the metacommunity is also fixed.

(iii) Simulate the dynamics of the local community: Simulate the dynamics of the local community by randomly replacing individuals in the local community. At each time step, individuals die at a given mortality rate, and empty cells due to deaths are randomly recolonized by new individuals. This process is similar to that for constructing the initial local community. That is, if an empty cell belongs to the $i$ th niche,

I, Determine if the next individual to fill this cell is whether an immigrant from the metacommunity or a local birth within the local community. If the former case is chose with probability $m$, go to [II], otherwise go to [III].

II, Pick a random individual from the metacommunity. Let $j$ be the species number of the chosen individual. Fill the cell if $q_{i, j}=1$, otherwise repeat this step until the cell is filled.

III, Pick a random individual from the local community. Let $j$ be the species number of the chosen individual. Fill the cell if $q_{i, j}=1$, otherwise repeat this step until the cell is filled. It should be noted that although very rare, there could be situations where this procedure does not work because any of all other individuals in the local community cannot survive in this niche (i.e., $q_{i, j}=0$ for all individuals in the local 
community). In such a case, the cell may be filled by an immigrant from the metacommunity. That is, go to [II].

\section{Simulations}

In our simulation, we assume $J=10,000$, and $J_{M}=10,000,000$. A single run of simulation consists of 20,000 time steps with a mortality rate of $1 \%$ per step, which are based on previous studies of neutral models in tropical forest (Condit et al., 2006). We set $\theta=50$ and $m=0.1$, which are very close to the estimates in tropical forests under neutral model (Etienne, 2005). We consider five different numbers of niches, $N=\left\{1,5,10,100, N_{\max }\right\}$, where $N_{\max }$ is the potentially maximum number of niches, which is identical to $S_{M}$. Note that $S_{M}$ is a variable that is determined by $\theta$ and $J_{M}$. For example, if $\theta=50$ and $J_{M}=10,000,000$ are given, $S_{M}$ would be an integer around 650 . Suppose $S_{M}$ is randomly determined to be 652 in step (i), then we assumed $N_{\max }=652$ when we analyzed the result of this replication. This treatment is commonly used in the previous neutral model simulation studies (Hubbell, 2001). For $p$, we used four values, $p=\{0,0.1,0.5,0.8\}$, in addition to the completely neutral case, $p=1$. In this work, simulations were performed for all pairs of these values of $p$ and $N$, except for $(p, N)=(0,1)$ because this is obviously a meaningless parameter set, i.e., the case where the community is composed of only one species with 10,000 individuals.

\section{Model selection}

A common approach to test Hubbell's neutral model is to compare the goodness-of-fit between the neutral model and other alternative models, e.g., by using AIC (Akaike's Information Criterion Akaike (1973)). A lognormal distribution (Preston, 1948) and a logseries distribution (Fisher et al., 1943) 
have been commonly used to represent non-neutral cases. We also include our niche model as an alternative, so that we select the best fit model among Hubbell's neutral model v.s. the three non-neutral models, i.e., it represents lognormal, logseries and our niche models. We below explain how these four models are fit to an observed SAD, which is generated by simulations as described in the previous section. Note that AIC can be suitable for model selection among nested models (i.e., simpler cases are special cases of more complex models) although it is commonly used in ecology to compare nonnested models (Johnson and Omland, 2004). In this sense, our niche model is one of very few alternative models in which Hubbell's neutral model is nested. Therefore, it is possible to statistically compare these two models with the AIC approach, or with more sophisticated likelihood-based methods (see the Discussion for more details). Nevertheless, in order to include all three alternative models, we here use the conventional AIC-based approach (i.e., non-nested cases).

To evaluate the performance of this model selection approach, we simulated a large number of SADs under our niche model, and investigated which model is selected for each SAD. In practice, given a simulated SAD, the four models were fitted and the maximum log-likelihoods were computed. Although there are a number of methods and software to estimate the best-fit parameters, in this work, it was needed to modify them in order to make a statistically fair comparison of the likelihoods for the four models. Because it is not possible to obtain a reliable expected SAD under our niche model (particularly for very rare abundance due to a lack of analytical expression), we had to use a binned SAD (see below for details). Therefore, to be consis- 

as

$$
L L=\sum_{r}\left\{n_{r} \log \left(\frac{E_{r}}{S}\right)\right\}
$$

$$
L L^{\prime}=\sum_{r}\left\{n_{r} \log \left(\frac{E_{r}}{n_{r}}\right)-\left[E_{r}-n_{r}\right]\right\}
$$

where $n_{r}$ and $E_{r}$ are the observed number of species and the expected number of species in the $r$ th abundance bin, respectively. $S$ is the total number of species. The expected SAD can be computed either analytically or by a simulation using the estimated parameter in each model (see below for each model). We searched the parameter set that maximizes the likelihood for all four models. In the following, we detail this process for each of the four models.

Hubbell's neutral model: Under Hubbell's neutral model, the expected binned SAD is given as a function of $\theta$ and $m$ ( $J$ is assumed to be known here). Because there is no adequate analytical expressions of SAD (but see Volkov et al., 2003), for a pair of $\theta$ and $m$, the expected binned SAD was obtained by averaging over 100 independent artificial species-abundance configurations by using the program of Etienne (2007).

The best-fit parameters were searched in wide parameter ranges: $\theta=$ $\{1,2,3, \ldots, 300\}$ and $m=\{0.05,0.1,0.15, \ldots, 1\}$ using the likelihood function:

(Kempton and Taylor, 1974; Hubbell, 2001). We confirmed that the best-fit 
$\theta$ and $m$ are almost identical to their maximum likelihood estimates obtained by using the method based on the Ewens sampling formula implemented by Etienne (2005). Using the best-fit parameter set, the likelihood of the binned SAD was re-calculated by (6) for subsequent model selection.

Niche model: This model has four parameters, $\theta, m, N$ and $p$. For a parameter set, the expected binned SAD was obtained by averaging over 100 independently simulated SADs (20,000 time steps for each replication. see the Niche Model section for details), and the best-fit parameters set was searched by (7) in wide ranges of the four parameters: $\theta=\{1,2,3, \ldots, 300\}, m=$ $\{0.05,0.1,0.15, \ldots, 1\}, N=\left\{1,5,10,100, N_{\max }\right\}$ and $p=\{0(N \neq 1), 0.1,0.5,0.8\}$. Using the best-fit parameter set, the likelihood of the binned SAD was calculated by (6) for model selection.

Lognormal function: It has traditionally been known that there are occasions in which SAD can be well approximated by a lognormal distribution, for example in a community under many ecological factors or a community with multidimensional resource utilization (May, 1975; Magurran, 2004). A lognormal distribution can be specified by with two parameters, the mean and variance. To fit a lognormal distribution to an observed SAD, it is needed to search for the best-fit parameters. To do so, it is common to use a SAD binned in Preston's octave (O'Hara and Oksanen, 2003), to which a generalized linear regression model with a standard lognormal distribution or a Poisson lognormal distribution is fitted. (\#3-5) Here, we employ a standard lognormal because it shows a better fit to our model than a Poisson lognormal. It is known that this method provides the identical estimates to the maximum likelihood method. After estimating the mean and variance of the 
lognormal distribution, we computed the log-likelihood of the binned SAD by (6).

Logseries function: A logseries distribution could approximate a typical SAD in (i) a community where the dynamics is simply dominated by one/a few ecological factors, (ii) a community where dominant species preempt the major part of the limited resource or (iii) a community that is not in equilibrium (May, 1975; Magurran, 2004). It should be noted that a logseries distribution is usually applied to an open community, although Hubbell's neutral SAD and lognormal distribution consider a fully-censused or closed community. Nevertheless, we here apply a logseries distribution to the "closed" local community because a closed community can be considered to be a special case of a subsampled or open community. Fitting a logseries distribution involves estimating two parameters, Fisher's $\alpha$ and $x$ (Fisher et al., 1943). For a pair of $\alpha$ and $x$, the expected binned SAD was numerically obtained, and the log-likelihood of the binned SAD was computed by (6). The best-fit parameter pair was searched in wide ranges of $\alpha$ and $x$ : $\alpha=\{1.00,1.01,1.02, \ldots, 200\}$ and $x=\{0.9500,0.9501,0.9502, \ldots, 1\}$.

Performance of neutrality tests

The performance of several neutrality tests are compared by applying them to simulated data. We consider Etienne's exact test (Etienne, 2007; Etienne and Rosindell, 2011) and other summary statistic-based tests as summarized in Table 1. Etienne's exact test can be considered as a summary statistic-based test because the likelihood of the configuration is treated as a summary statistic. 
Given a set of simulated data, we computed the summary statistics in Table 1, and their P-values were evaluated. For this, we obtained the distributions of the summary statistics, which was created by randomly generating data (1,000 replications) conditional on the maximum likelihood estimates of $\hat{\theta}$ and $\hat{m}$. We here define the P-value as the proportion of replications that rejected Hubbell's neutral model conditional on $\hat{\theta}$ and $\hat{m}$. This procedure is shared by all summary statistic-based tests in Table 1 as described below (also see Appendix Fig. A1).

I, Simulate data to be tested. The data are denoted by $\mathcal{D}$, which is the configuration of species abundance. Then, compute the summary statistic $(S S)$ of interest for $\mathcal{D}$, which is denoted by $S S_{\mathcal{D}}$.

II, Determine the P-value for $S S$. First, estimate $\theta$ and $m$ from $\mathcal{D}$ using the maximum likelihood method based on the Ewens sampling formula implemented in the PARI/gp program by Etienne (2005). Note that $J$ is treated as known because we consider a closed local community, that is, we have data for all individuals in the community. The estimated parameters are denoted by $\hat{\theta}$ and $\hat{m}$. Then, conditional on $\hat{\theta}$ and $\hat{m}$, we independently generate 1,000 realizations of species-abundance configuration under Hubbell's neutral model according Etienne's algorithms implemented in the PARI/gp program (Etienne, 2007, 2005). For each random configuration, we calculate various summary statistics (see Table 1).

III, Determine the P-value. Etienne's exact test is treated as a one-tailed test, while all the others are two-tailed tests. Let $r$ be the proportion 
of the simulation runs with $S S$ (or likelihood) less than $S S_{\mathcal{D}}$. Then, the P-value for Etienne's exact test is identical to $r$, while for the other two-tailed tests, the $\mathrm{P}$-value is $2 r$ if $r<0.5$ otherwise $1-2 r$.

For computing $S S_{\mathcal{D}}$ for Etienne's exact test (i.e., log-likelihood), the program of Etienne (2007) is used, while the vegan package in $\mathrm{R}$ is useful for some of the other tests. (\#3-6) We used it to calculate Shannon's H, Simpson's $D$, Fisher's $\alpha$ and rarefaction in this study. Again, we emphasize that this $\mathrm{P}$-value is conditional on $\hat{\theta}$ and $\hat{m}$, which cannot be considered as the real P-value of parameter-free neutral model. Because it is extremely difficult to obtain the unconditional P-value, this 'conditional' one has been used so often since the introduction of Hubbell's neutral model. Therefore, we follow this procedure, which may work at least for relative comparison of their performance, even when statistically incorrect.

\section{Results}

Our simulations clearly demonstrate that there is a strong effect of nichepreference on the pattern of species diversity (i.e., SAD and $S$ ) in the local community (Fig. 1. See also Fig. A2 for a plot of $S$ ). When $p=1$ (complete neutral case), the average $S$ is $197.8 \pm 5.5$ ( \pm SD), which is consistent with the expectation under Hubbell's neutral model. The other extreme case would be when $p=0$ and $N=1$, where all cells in the local community belong to one kind of niche and only one species is allowed to occupy the niche, so that there is only one species with abundance $J=10,000$. As the number of niches $(N)$ increases (but $p=0$ is fixed), the number of species $(S)$ increases to the theoretical maximum $(S=650$, which is approximate 
number of species in the metacommunity when $J_{M}=10,000,000$ and $\theta=50$ ). As $p$ increases (with $N$ fixed), the SAD becomes close to the neutral one. Thus, our model enables us to explore situations with various degrees of niche differentiation.

Our results demonstrate strong effects of niche differentiation on the SAD and the number of species. However, as mentioned in the Introduction, if we look at the SAD alone, the observed SAD is well fitted by Hubbell's neutral model visually (blue line, Fig. 1). This is in good agreement with previous studies which showed good fit of Hubbell's neutral model by eyes (Volkov et al., 2007; Chave et al., 2002; Hubbell, 2001). This good fit of Hubbell's neutral model is simply due to the estimated $\theta$ and $m$ that are far from the given values $(\hat{\theta}=50, \hat{m}=0.1)$ (especially for a small $p$, e.g. $(\hat{\theta}, \hat{m})=(5.8$, $0.2)$ for $(N, p)=(1,0.1)$ in Fig. 1 ; see also Fig. A3). It is found that a lognormal distribution also provides the best fit of observation for a wide range of $p$ and $N$, while the fit of a logseries distribution is not very good. This result is consistent with previous studies (Adler et al., 2007; Chave, 2004; Volkov et al., 2005; Bell, 2005) that pointed out that there would be no significant difference between the lognormal and Hubbell's neutral model. The major purpose of this work is to quantitatively evaluate this problem in model selection. We performed a number of simulations, and the results are summarized in Fig. 2A. Under the complete neutral model $(p=1)$, the observed SADs in 98 of the 100 replications are best explained by Hubbell's neutral model. The inferred parameters were $\hat{\theta} \sim 50$ and $\hat{m} \sim 0.1$, which were close to the given parameters. The pattern is not very different when $p=0.8$; the neutral model is best supported in $>\sim 60 \%$ of replications, and the fit 
of the lognormal distribution is the second best. When $N \geq 5$ and $p \leq 0.5$, either the lognormal or our niche model is best supported. The fit of our niche model is particularly good with very strong niche-structure (i.e., $N \geq 5$ and $p \leq 0.1$. Thus, when we use $\hat{\theta}$ and $\hat{m}$ estimated from the local community itself, unless the degree of niche preference is very strong (i.e., small $p$ and large $N$ ), it can be concluded that the fit of Hubbell's neutral model is quite good, and the power to reject Hubbell's neutral model is very limited. The major reason for this overfitting of Hubbell's neutral model is that we do not know the precise values of $\theta$ and $m$. To demonstrate this, we performed the same analysis by assuming we know the given values (i.e, $\theta=50$ and $m=0.1)$. Then, we found that the power to reject Hubbell's neutral model is substantially improved especially for intermediate values of $p$ (Fig. 2B).

Through this work, we used Preston's octave classes (Preston, 1948), which are $\log _{2}$-based bins (i.e., $\{1,2,3-4,5-8,9-16, \ldots\}$ ) with some adjustment at borders between adjacent bins. Although Preston's octave classes are commonly used, our result might change if we use other bins. To check the effect of bin sizes, we repeated the same analysis with two additional bins, normal $\log _{2}$ and $\log _{10}$. As shown in Appendix Fig. A4 and A5, we obtained essentially identical results to those with Preston's octave classes, except that the fit of our niche model is generally better.

We also explore the performance of other neutrality tests, namely, Etienne's exact test and the summary statistic-based tests summarized in Table 1. It should be noted that all these tests are usually performed with $\hat{\theta}$ and $\hat{m}$ estimated from the local community to be tested, so that they share the same problem of the SAD-fitting approach, but the extent of the 
sensitivity to this unknownness may differ depending on the test. Fig. 3A shows the P-values of all 10 tests when $\hat{\theta}$ and $\hat{m}$ are used. It is found that Hubbell's neutral model was rejected in almost all cases; when $p \geq 0.8$ or $N=1$, which is consistent with Hubbell's SAD fitting approach. Etienne's exact test, species richness, Fisher's $\alpha$ and rarefaction $J^{*} 0.5$ failed to reject Hubbell's neutral model in the most cases (except for Etienne's exact test when $p=0$ and $N=100,650)$. Rarefaction $J^{*} 0.1$ rejected Hubbell's neutral model only when the niche structure is strong, that is, $p$ is small and $N$ is large. On the other hand, Shannon's $H$, Simpson's $D$, inv $N$, inv $N_{2}$ and variance $N i$ performed better, suggesting that they are relatively sensitive to niche structure.

We also investigated how the performance of these tests can be improved if we know the given values of $\theta$ and $m$. As expected, Fig. 3B shows that their performance is substantially improved in comparison with Fig. 3A. Thus, it is again demonstrated the fact that we do not know the true value of $\theta$ and $m$ causes a reduction in the performance of the neutrality tests.

\section{Discussion}

It seems that there is a two-fold problem in testing neutrality in community ecology. First, there are a number of possible neutral models, but the best known one (i.e., Hubbell's neutral model) has been so well accepted and used widely as a representative neutral model. Therefore, rejecting Hubbell's neutral model does not necessarily mean that the neutrality is rejected. Second, in most cases, it is quite difficult to reject even the simplest neutral model with the current methods and data. The focus of this study is the second problem, the problem of current methods, because we cannot proceed 
without solving this technical problem. The first one will be a challenging problem in a next step, which is beyond the scope of this work.

In this study, we first developed a new niche model that incorporates stochastic demography of individuals together with the mechanism of niche differentiation as a deterministic factor. The model involves a pair of parameters, $p$ and $N$; the former represents the degree of niche preference and the latter is the number of different niches in the local community. Our niche model has a nested-structure with Hubbell's neutral model, which allows us to make a fair statistical comparison between two models and select the best model according to AIC. Furthermore, it is possible to use more statistically rigorous approaches, such as the likelihood ratio test. It should be noted that the AIC-based comparison of Hubbell's neutral model and the lognormal and the logseries distributions is not statistically correct, although because this method is very frequently used, we followed it in order to investigate the performance.

Another advantage of our model is that it allows one to explore various degrees of niche preference by changing $p$. When $p=1$, the model is identical to Hubbell's neutral model, while in the other extreme case with $p=0$, all species have their specific niches. We demonstrated that strong niche preference influences the pattern of species abundance (i.e., small $p$ ), showing quite different SADs from that expected under Hubbell's neutral model (i.e., $p=1) . S$ is affected by both $p$ and $N$. As shown in Fig. $1, S$ decrease as $p$ decreases. In the niche site, a dead individual is likely to be replaced by the species that are abundant in the same niche type or a generalist species. The preference of species in each niche would limit recruitment of rare species 
or specialists and induce a reduction of $S$. In other words, the level of niche overlap among species directly affects the neutrality of the community, thereby reducing $S$. With increasing $N, S$ increases (Fig. A2 ). When there are a large number of niches in the local community, even if each niche has strong species-preference, $S$ is not reduced.

Our niche model was used to evaluate the performance of various tests of neutrality (or Hubbell's neutral model). We found that all neutrality tests we used here did not always perform very well (Figs. 2A and 3A). This is simply because the most important parameters $(\theta$ and $m)$ to characterize the metacommunity that provides the basis of the local community are unknown, so that we have to estimate them from the local community to be tested. Such a conventional treatment likely causes an overfitting. This overfitting problem has been repeatedly pointed out for Hubbell's SAD-fitting approach by many authors (Chave, 2004; Chisholm and Pacala, 2010; Volkov et al., 2005), but it should also apply to fitting other models (or distributions). We here investigated the effect of this problem on the performance of neutrality tests quantitatively. As we showed in Figs. 2B and 3B, the performance was substantially improved if we know the true values of $\theta$ and $m$, indicating the importance of having better estimates of $\theta$ and $m$.

Thus, our results suggest that for improving the performance, we need (i) to develop new methods which are more robust to unknown $\theta$ and $m$, or (ii) to estimate $\theta$ and $m$ from data that are independent from the local community to be tested. For (i), along the line of the model-fitting approach, we probably need more options for alternative distributions, in addition to the commonly used lognormal and logseries ones. We emphasize this because 
occasionally these two distributions alone are not sufficient to reject the null neutral model but other mechanistic models can. Indeed, in our simulation, there are a number of replications where our niche model exhibited the best fit (Fig. 2A). It is suggested that if more alternative distributions were available, the performance to reject the null neutral model would be significantly better. For summary statistic-based tests, it is desired to develop new summary statistics for example, the one elegantly summarized all information of the species abundance such as species richness, evenness and abundance of rare species. Moreover, those that are robust to $\theta$ and $m$ are preferred.

It may be more powerful if we can solve problem (ii). Unfortunately, it would be extremely difficult to estimate $\theta$ and $m$ from data that are independent from the local community to be tested. Ideally, $\theta$ and $m$ should be estimated from the metacommunity, accurately delimiting and sampling the metacommunity is extremely difficult especially when its scale is unknown. It may also be very difficult to use other kinds of data, from which $\theta$ and $m$ can be estimated (but see the work by Chisholm and Lichstein (2009), which estimated one of the parameters, $m$, from dispersal data in a local community). Then, what can we do when such independent estimates of $\theta$ and $m$ are not available? A possibility is to use multiple data sets that should share the same (or at least similar) values of $\theta$ and $m$. For example, suppose here are multiple local communities that share a single metacommunity. This is not an unrealistic situation and was suggested by Etienne et al. (2007). Comparing these multiple local communities could provide much more information not only on $\theta$ and $m$ in the shared metacommunity but also the mechanisms that shaped the observed species richness and abundance in each local com- 
munity. It would be also very powerful to have data at multiple time points even in a single local community (Etienne et al., 2007; Tsai et al., 2014; Magurran, 2007; McGill et al., 2007). In addition, any kind of community dynamics data through field observations over multiple years would be informative. Examples include information on which individual was replaced by which individual at what time point. Together with such multidimensional data, development of statistical methods to analyze them is needed.

In summary, our niche model and simulation provided insight into how to understand the observed SADs and their fitting to models. We quantitatively demonstrated that it is very difficult to reject Hubbell's neutral model from SAD alone, and suggested several ideas to solve this problem (at least partially). hlWhile the assumptions of Hubbell' s neutral model are too simplistic for some ecologists to accept intuitively, the neutral model can be used as null model as it is a good approximation to a neutral community structure (Rosindell et al., 2012). The important role of his neutral model should be as a null model to be tested, and its rejection indicates that some kind of non-neutral processes should be involved and that models incorporating such processes could lead to a better understanding of the mechanisms shaping the configuration of the community. In this sense, we would like to again emphasize the importance of developing statistical methods with much higher power than those currently available.

\section{Acknowledgements}

We are grateful to J. Rosindell, anonymous reviewers and the members of Innan Lab for valuable comments and discussion on an earlier version of the manuscript. The authors declare no conflict of interest. 


\section{References}

Adler, P. B., Hillerislambers, J. and Levine, J. M. 2007. A niche for neutrality. Ecology letters 10: 95-104.

Akaike, H. 1973. Information theory as an extension of the maximum likelihood principle. In: Petrov, B. N. and Caski, F. (eds.), Proceedings of the 2nd International Symposium on Information Theory, pp. 267-281. Akadimiai Kiado, Budapest.

Alonso, D., Etienne, R. S. and McKane, A. J. 2006. The merits of neutral theory. Trends In Ecology \& Evolution 21: 451-457.

Bell, G. 2005. The co-distribution of species in relation to the neutral theory of community ecology. Ecology 86: 1757-1770.

Caswell, H. 1976. Community structure: A neutral model analysis. Ecological Monographs 46: 327-354.

Chave, J. 2004. Neutral theory and community ecology. Ecology Letters 7: $241-253$.

Chave, J., Muller-Landau, H. and Levin, S. 2002. Comparing classical community models: Theoretical consequences for patterns of diversity. American Naturalist 159: 1-23.

Chesson, P. 2000. Mechanisms of maintenance of species diversity. Annual Review of Ecology and Systematics 31: 343-366.

Chisholm, R. A. and Lichstein, J. W. 2009. Linking dispersal, immigration and scale in the neutral theory of biodiversity. Ecology letters 12: 1385-93. 
Chisholm, R. A. and Pacala, S. W. 2010. Niche and neutral models predict asymptotically equivalent species abundance distributions in high-diversity ecological communities. Proceedings of the National Academy of Sciences of the United States of America 107: 15821-15825.

Clark, J. S. 2012. The coherence problem with the unified neutral theory of biodiversity. Trends In Ecology \& Evolution 27: 198-202.

Condit, R., Ashton, P., Bunyavejchewin, S., Dattaraja, H. S., Davies, S., Esufali, S., Ewango, C., Foster, R., Gunatilleke, I. A. U. N., Gunatilleke, C. V. S., Hall, P., Harms, K. E., Hart, T., Hernandez, C., Hubbell, S., Itoh, A., Kiratiprayoon, S., Lafrankie, J., de Lao, S. L., Makana, J.-R., Noor, M. N. S., Kassim, A. R., Russo, S., Sukumar, R., Samper, C., Suresh, H. S., Tan, S., Thomas, S., Valencia, R., Vallejo, M., Villa, G. and Zillio, T. 2006. The importance of demographic niches to tree diversity. Science 313: 98-101.

Dornelas, M., Connolly, S. R. and Hughes, T. P. 2006. Coral reef diversity refutes the neutral theory of biodiversity. Nature 440: 80-82.

Engen, S. and Lande, R. 1996. Population dynamic models generating the lognormal species abundance distribution. Mathematical Biosciences 132: 169-183.

Etienne, R. 2005. A new sampling formula for neutral biodiversity. Ecology Letters 8: 253-260.

Etienne, R. and Olff, H. 2005. Confronting different models of community 
structure to species-abundance data: a bayesian model comparison. Ecology Letters 8: 493-504.

Etienne, R. S. 2007. A neutral sampling formula for multiple samples and an 'exact' test of neutrality. Ecology letters 10: 608-18.

Etienne, R. S. and Alonso, D. 2007. Neutral community theory: How stochasticity and dispersal-limitation can explain species coexistence. Journal of Statistical Physics 128: 485-510.

Etienne, R. S., Alonso, D. and McKane, A. J. 2007. The zero-sum assumption in neutral biodiversity theory. Journal of theoretical biology 248: 522-36.

Etienne, R. S. and Rosindell, J. 2011. The spatial limitations of current neutral models of biodiversity. PLoS One 6: e14717.

Ewens, W. J. 1972. The sampling theory of selectively neutral alleles. Theor Popul Biol 3: 87-112.

Fisher, R., Corbet, S. and Williams, C. 1943. The relation between the number of species and the number of individuals in a random sample of an animal population. Journal of Animal Ecology 12: 42-58.

Gotelli, N. J. and McGill, B. J. 2006. Null versus neutral models: what's the difference? Ecography 29: 793-800.

Gravel, D., Canham, C. D., Beaudet, M. and Messier, C. 2006. Reconciling niche and neutrality: the continuum hypothesis. Ecology Letters 9: 399409. 
Harpole, S. W. and Tilman, D. 2006. Non-neutral patterns of species abundance in grassland communities. Ecology Letters 9: 15-23.

He, F. 2005. Deriving a neutral model of species abundance from fundamental mechanisms of population dynamics. Functional Ecology 19: 187-193.

Hubbell, S. P. 2001. The unified neutral theory of biodiversity and biogeography, volume 32. Princeton University Press, Princeton.

Innan, H., Zhang, K., Marjoram, P., Tavaré, S. and Rosenberg, N. A. 2005. Statistical tests of the coalescent model based on the haplotype frequency distribution and the number of segregating sites. Genetics 169: 1763-1777.

Jabot, F. and Chave, J. 2009. Inferring the parameters of the neutral theory of biodiversity using phylogenetic information and implications for tropical forests. Ecology Letters 12: 239-248.

Jabot, F. and Chave, J. 2011. Analyzing tropical forest tree species abundance distributions using a nonneutral model and through approximate bayesian inference. The American naturalist 178: E37-47.

Johnson, J. and Omland, K. 2004. Model selection in ecology and evolution. Trends In Ecology \& Evolution 19: 101-108.

Kempton, R. and Taylor, L. 1974. Log-series and log-normal parameters as diversity discriminants for the lepidoptera. Jornal of Animal Ecology 43: $381-399$.

Lande, R., Engen, S. and Sæther, B.-E. 2003. Stochastic population dynamics in ecology and conservation. Oxford University Press, Oxford. 
Locey, K. J. and White, E. P. 2013. How species richness and total abundance constrain the distribution of abundance. Ecol Lett 16: 1177-85.

Magurran, A. 2004. Measuring Biological Diversity. Blackwell Science LTD, Oxford, first edition.

Magurran, A. E. 2007. Species abundance distributions over time. Ecol Lett 10: $347-54$.

Matthews, T. J. and Whittaker, R. J. 2014. Neutral theory and the species abundance distribution: recent developments and prospects for unifying niche and neutral perspectives. Ecology and Evolution 4: 2263-2277.

May, R. M. 1975. Patterns of species abundance and diversity. In: Cody, M. L. and Diamond, J. M. (eds.), Ecology and evolution of communities, pp. 81-120. Belknap Press of Harvard University Press, Cambridge, Mass.

McGill, B. J., Etienne, R. S., Gray, J. S., Alonso, D., Anderson, M. J., Benecha, H. K., Dornelas, M., Enquist, B. J., Green, J. L., He, F., Hurlbert, A. H., Magurran, A. E., Marquet, P. A., Maurer, B. A., Ostling, A., Soykan, C. U., Ugland, K. I. and White, E. P. 2007. Species abundance distributions: moving beyond single prediction theories to integration within an ecological framework. Ecology letters 10: 995-1015.

O'Hara, R. and Oksanen, J. 2003. fisherfit vegan Fit Fisher's logseries and Preston's lognormal model to abundance data. http://cc.oulu.fi/ jarioksa/softhelp/vegan/html/fisherfit.html.

Preston, F. 1962. The canonical distribution of commonness and rarity: Part i. Ecology 43: 185-215. 
Preston, F. W. 1948. The commonness, and rarity, of species. Ecology 29: $254-283$

Purves, D. W. and Pacala, S. W. 2005. Ecological drift in niche-structured communities:neutral pattern does not imply neutral process. In: Burslem, D. F. R. P., Pinard, M. A. and Hartley, S. E. (eds.), Biotic interactions in the Tropics: their role in the maintenance of species diversity, pp. 107-136. Cambridge University Press, Cambridge.

Rosindell, J., Hubbell, S. P., He, F., Harmon, L. J. and Etienne, R. S. 2012. The case for ecological neutral theory. Trends In Ecology \& Evolution 27: 203-208.

Rosindell, J., Wong, Y. and Etienne, R. S. 2008. A coalescence approach to spatial neutral ecology. Ecological Informatics 3: 259 - 271.

Slatkin, M. 1994. An exact test for neutrality based on the ewens sampling distribution. Genetical research 64: 71-74.

Slatkin, M. 1996. A correction to the exact test based on the ewens sampling distribution. Genetical research 68: 259-260.

Sugihara, G. 1980. Minimal Community Structure: An Explanation of Species Abundance Patterns. American Naturalist 116: 770-787.

Sugihara, G., Bersier, L.-F., Southwood, T. R. E., Pimm, S. L. and May, R. M. 2003. Predicted correspondence between species abundances and dendrograms of niche similarities. Proceedings of the National Academy of Sciences of the United States of America 100: 5246-5251. 
Tilman, D. 1982. Resource competition and community structure, volume 17. Princeton University Press, Princeton.

Tilman, D. 2004. Niche tradeoffs, neutrality, and community structure: A stochastic theory of resource competition, invasion, and community assembly. Proceedings of the National Academy of Sciences of the United States of America 101: 10854-10861.

Tokeshi, M. 1990. Niche apportionment or random assortment: Species abundance patterns revisited. Journal of Animal Ecology 59: 1129-1146.

Tokeshi, M. 1992. Dynamics of distribution animal communities - theory and analysis. Researches On Population Ecology 34: 249-273.

Tsai, C.-H., Miki, T., Chang, C.-W., Ishikawa, K., Ichise, S., Kumagai, M. and Hsieh, C.-h. 2014. Phytoplankton functional group dynamics explain species abundance distribution in a directionally changing environment. Ecology .

Ulrich, W., Ollik, M. and Ugland, K. I. 2010. A meta-analysis of speciesabundance distributions. Oikos 119: 1149-1155.

Volkov, I., Banavar, J. R., He, F., Hubbell, S. P. and Maritan, A. 2005. Density dependence explains tree species abundance and diversity in tropical forests. Nature 438: 658-61.

Volkov, I., Banavar, J. R., Hubbell, S. P. and Maritan, A. 2003. Neutral theory and relative species abundance in ecology. Nature 424: 1035-7. 
${ }_{817}$ Volkov, I., Banavar, J. R., Hubbell, S. P. and Maritan, A. 2007. Patterns ${ }_{818}$ of relative species abundance in rainforests and coral reefs. Nature 450: $819 \quad 45-9$.

${ }_{820}$ Westoby, M., Falster, D., Moles, A., Vesk, P. and Wright, I. 2002. Plant eco821 logical strategies: Some leading dimensions of variation between species. 822 Annual Review of Ecology and Systematics 33: 125-159.

${ }_{823}$ Wright, S. 2002. Plant diversity in tropical forests: a review of mechanisms 824 of species coexistence. Oecologia 130: 1-14.

825 
839

\section{Figure legends}

Fig. 1 - The expected SADs of the niche model with various pairs of $p$ and $N$. The expected SAD was obtained by averaging over 100 replications of simulation. Error bars represent SD. Four models (Hubbell's neutral, lognormal, logseries and our niche model) were fit to each average SAD. The estimated $\theta$ and $m$ for fitting Hubbell's neutral model are shown in blue, hllognormal in yellow, logseries in orange, and our niche model in red.

Fig. 2 - Summary of the model selection by AIC. The proportions of simulation runs that support neutral model, lognormal, logseries, and our niche model are shown in different colors. (A) Results when estimated $\theta$ and $m$ are used. (B) Results when we know the actual parameter $\theta=50$ and $m=0.1$. See text for details.

Fig. 3 - Summary of the Etienne's exact test (1) and neutrality tests based on nine summary statistics (2-10). The proportions of simulation runs that rejected Hubbell's neutral mode are shown in orange, otherwise in blue. (A) Results when estimated $\theta$ and $m$ are used. (B) Results when we know the actual parameter $\theta=50$ and $m=0.1$. 


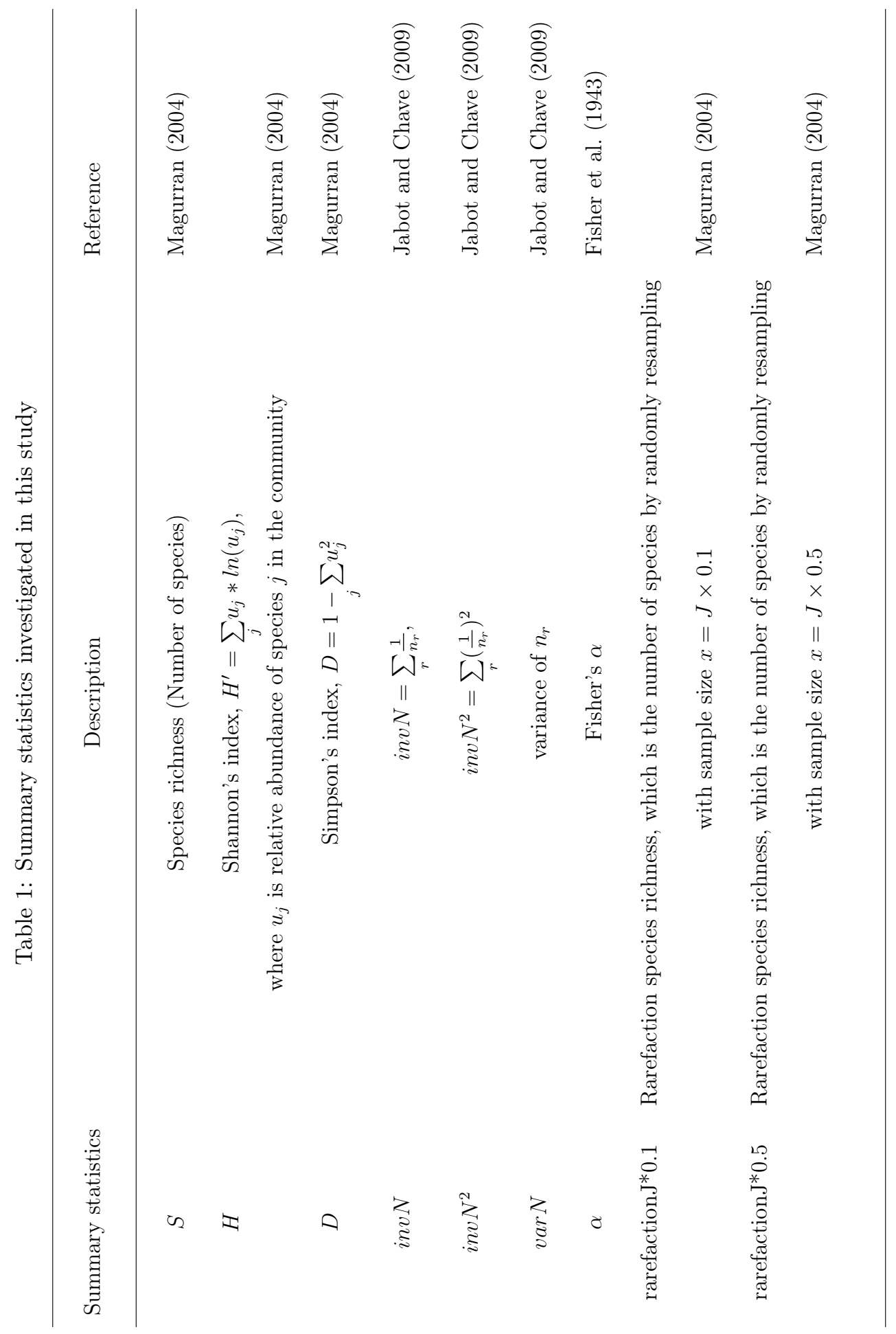



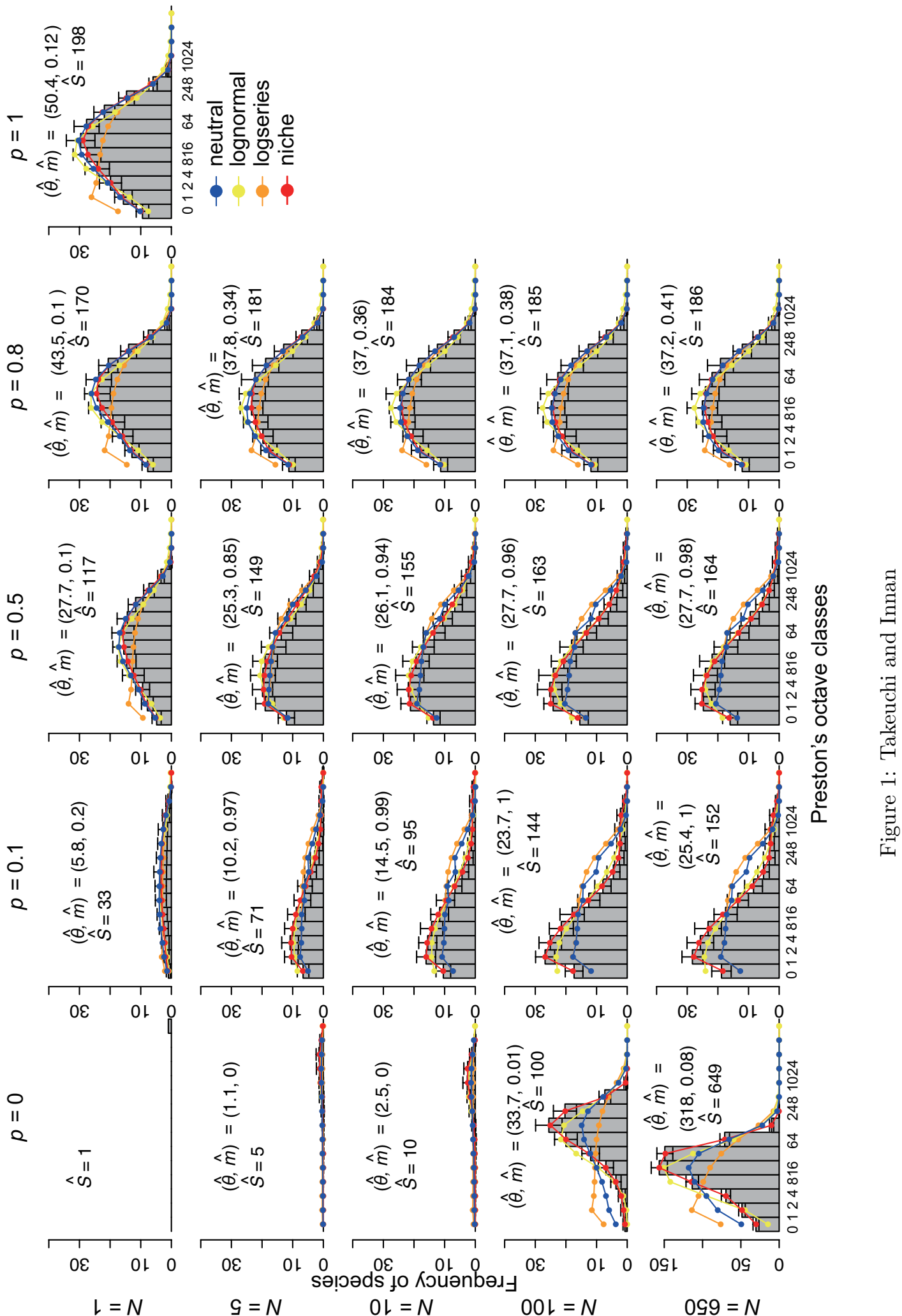


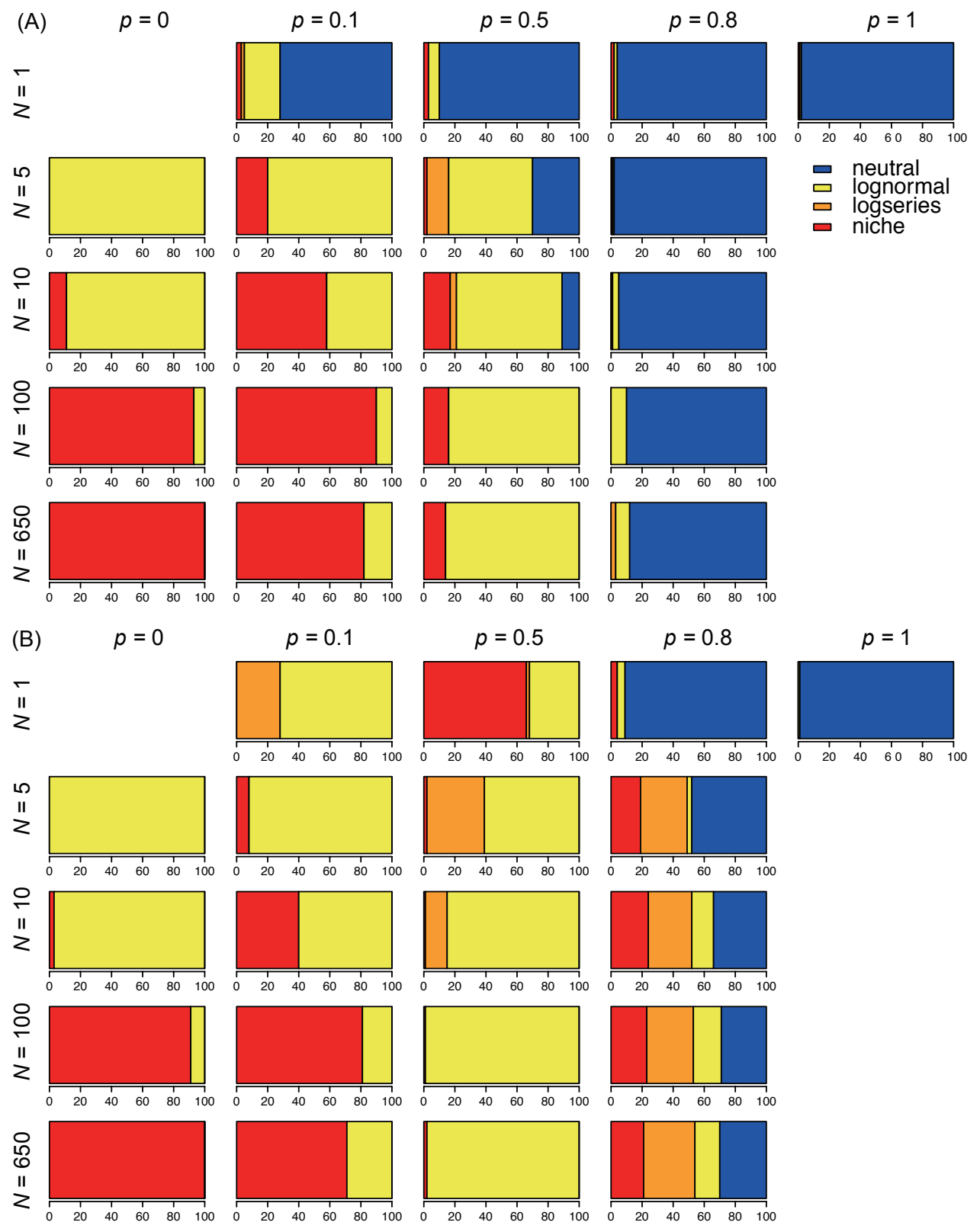

Figure 2: Takeuchi and Innan 


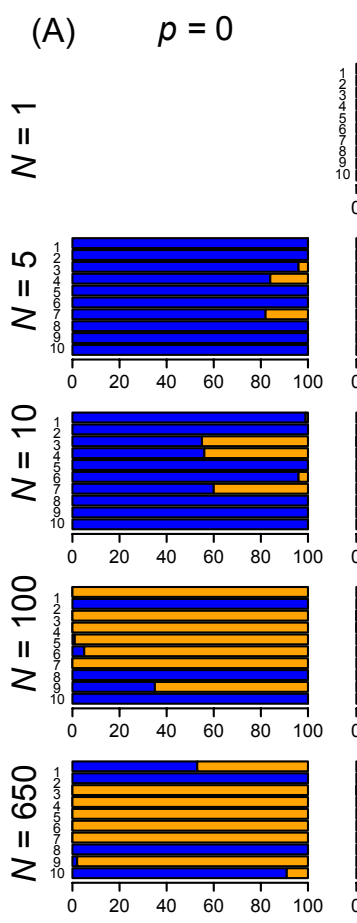

$p=0.1$
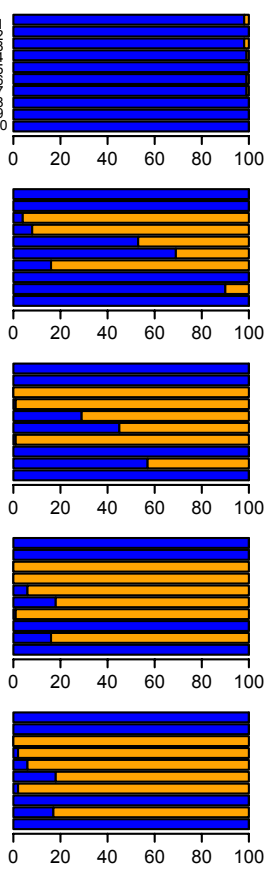

(B) $\quad p=0$

$$
\stackrel{5}{21}
$$
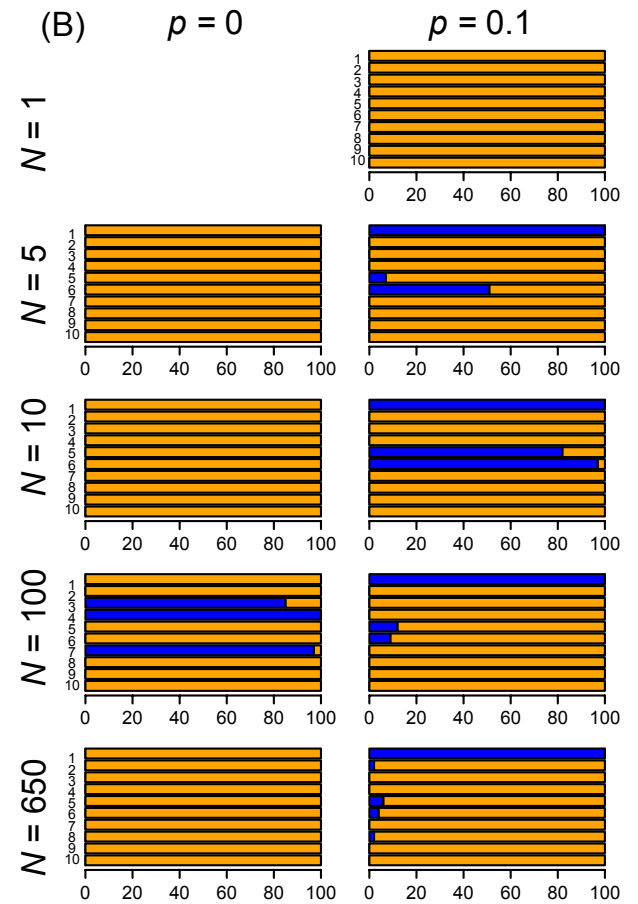

$p=0.5$
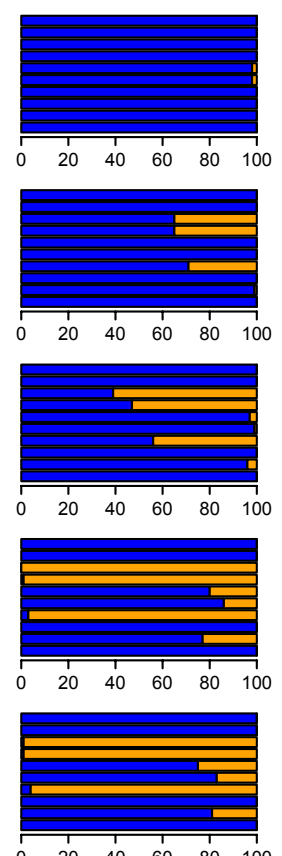

$p=0.5$
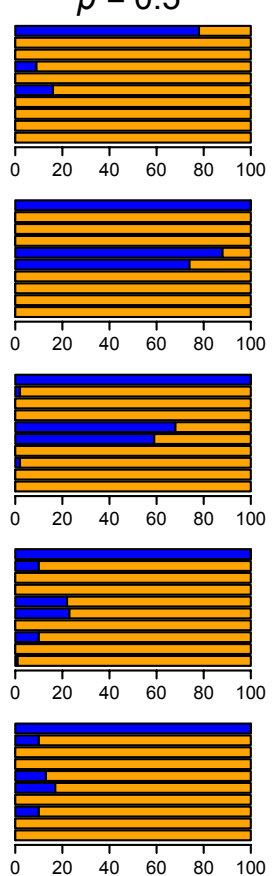

$p=0.8$

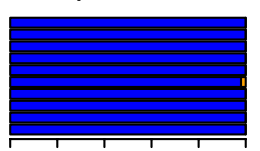

\begin{tabular}{lllllllllll}
\hline & 1 & 1 & 1 & 1 & 1 & 1 & 1 & 1 & 1 & 10 \\
20 & 40 & 60 & 80 & 100 & 0 & 20 & 40 & 60 & 80 & 100
\end{tabular}
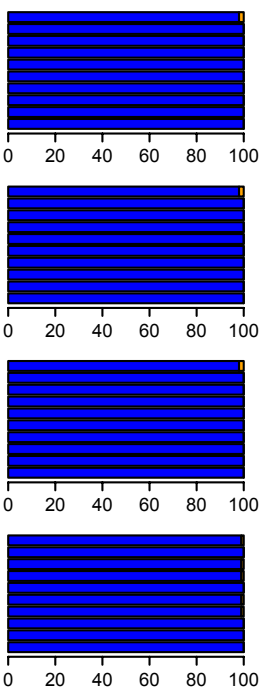

$p=0.8$
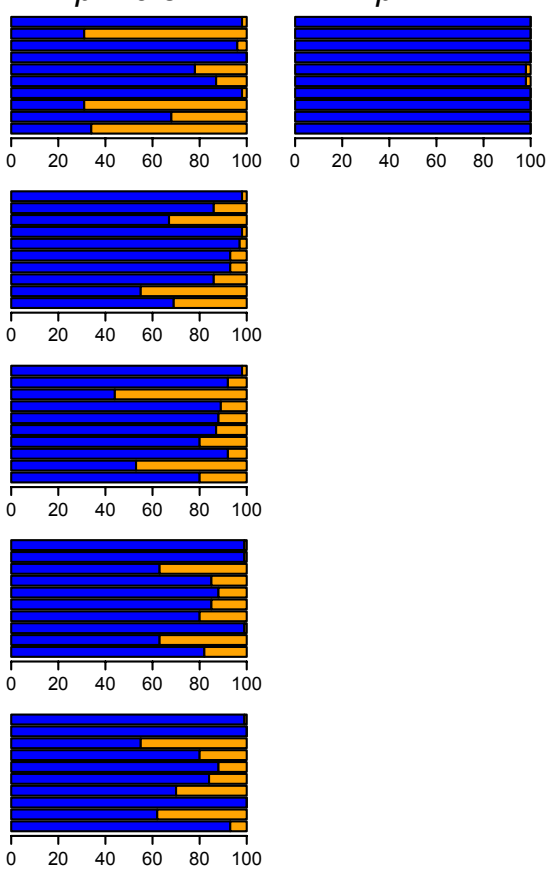

$\square$ not reject

$\square$ reject

1 Etienne's exact test

2 Species richness

3 Shannon's H

4 Simpson's D

5 inverse $\mathrm{Ni}$

6 variance $\mathrm{Ni}$

7 inverse $\mathrm{Ni} 2$

8 fisher's alpha

9 rarefaction $\mathrm{J}^{*} 0.1$

10 rarefaction $\mathrm{J}^{\star} 0.5$

Figure 3: Takeuchi and Innan

43 